\title{
Biosynthesis of amycolamicin: the biosynthetic origin of a branched $\alpha$-aminoethyl moiety in the unusual sugar amycolose
}

The Journal of Antibiotics (2010) 63, 147-149; doi:10.1038/ja.2010.1; published online 29 January 2010

A mycolamicin (Figure 1, 1) is a new antibiotic, which has been isolated from the culture broth of Amycolatopsis sp. MK575-fF5 during our screening program for drug-resistant pathogens. ${ }^{1,2}$ Structurally, 1 has unique features compared with other antibiotics; it contains trans-decalin, tetramic acid, two unusual sugars and pyrrole carbonic acid. 1 has bactericidal activity not only against a wide variety of bacteria but also against drug-resistant pathogens, methicillin-resistant Staphylococcus aureus (MIC90: 0.39 $\mu \mathrm{g} \mathrm{ml}^{-1}$ ) and vancomycin-resistant enterococci (MIC90: 0.2-0.78 $\mu \mathrm{g} \mathrm{ml}^{-1}$ ), which are major pathogenic bacterial causes of hospital infections. ${ }^{3}$ There is a strong need to develop novel antibiotics with unique structures and modes of action because pathogens resistant to the 'last resort' antibiotic vancomycin have emerged. ${ }^{4}$ Judging from the present situation, amycolamicin could be considered as a potential candidate antibiotic.

Unusual sugars have often been found in the structures of bioactive natural products and have important roles in expressing their activities. ${ }^{5-7}$ Amycolamicin has two unusual sugars, one is a 6-deoxyallose derivative, which is connected by an $\mathrm{N}$-glycoside bond through the tetramic acid, and the other is a novel sugar, $(3 R, 4 R, 5 R)-3$ - $\alpha$-aminoethyl-2,6dideoxy-hexopyranose, named amycolose (Figure 1,2), tethered by $O$-glycoside through the decalin ring. Both of these sugars are thought to be important for the antimicrobial activity of amycolamicin (unpublished data). Generally, two-carbon branched-chain sugars have been found to be $\alpha$-oxoethyl or $\alpha$-hydroxyethyl groups in nature, such as the glycosidic components of secondary metabolites. ${ }^{8,9}$ However, this is the first finding of a new sugar with a branched $\alpha$-aminoethyl moiety in the structure of amycolose at the C-3 position.

The biosynthesis of branched-chain sugars has been studied extensively. ${ }^{8,10-12}$ These main sugar skeletons originate in glucose 1-phosphate, similar to other unusual sugars. The one-carbon branch is derived from $S$-adenosylmethionine, but less is known about the two-carbon branch. The twocarbon branch may be biosynthesized from pyruvate, which has been studied using isotope tracer experiments, ${ }^{13-15}$ enzyme reactions $^{16}$ and gene inactivations. ${ }^{17}$ However, these modifications for the two-carbon branched chain are restricted to the $\alpha$ oxoethyl or $\alpha$-hydroxyethyl branch, and nothing has been reported for the $\alpha$-aminoethyl branch. In this paper, we report the origin of a branched $\alpha$-aminoethyl moiety, which we investigated with a feeding experiment that uses the stable-isotope-labeled pyruvate.

A tracer experiment that uses a ${ }^{13} \mathrm{C}$-labeled compound is an effective tool for revealing biosynthetic precursors. The ${ }^{13} \mathrm{C}$-label is detectable by NMR, and, especially in the case of contiguous labels, spin coupling is observed and intact incorporation can be easily clarified. Therefore, the feeding of $\left[2,3-{ }^{13} \mathrm{C}_{2}\right]$ pyruvate was carried out. Fermentation was done under conditions known to produce the desired results: a $500-\mathrm{ml}$ baffled flask containing $100 \mathrm{ml}$ production medium consisting of $0.5 \%$ glycerol, $0.5 \%$ dextrin, $0.2 \%$ soy bean meal, $0.05 \%$ yeast extract, $0.2 \% \mathrm{NaCl}, 0.05 \%$ $\mathrm{MgSO}_{4} \cdot 7 \mathrm{H}_{2} \mathrm{O}, 0.05 \%\left(\mathrm{NH}_{4}\right)_{2} \mathrm{SO}_{4}, 0.1 \%$ trace element solution and $0.1 \% \mathrm{CaCO}_{3}$ was incubated at $28{ }^{\circ} \mathrm{C}$ with shaking at 180 r.p.m. on a rotary shaker. Feeding of labeled sodium pyruvate ( $1 \mathrm{~g}$ ) was carried out 2 days after inoculation into the culture broth (11), and fermentation was continued for 2 days. The resulting fermentation broth was centrifuged, and the supernatant was extracted with ethyl acetate. The resulting crude extract was purified by centrifugal partition chromatography (CPC240, Senshu Scientific Co., Suginami-ku, Tokyo, Japan) with ${ }^{t} \mathrm{BuOMe:MeCN}: 0.1 \mathrm{M}$ $\mathrm{NaHCO}_{3}$ in an ascending mode. The fractions containing amycolamicin were further

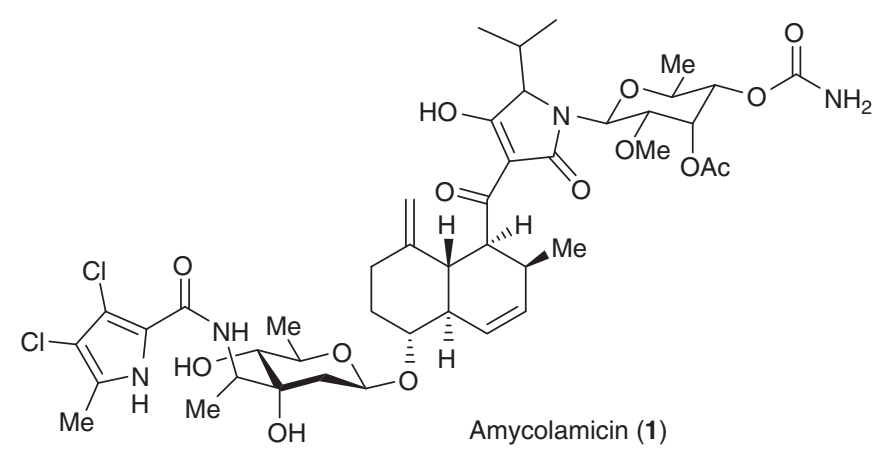

Figure 1 Structures of amycolamicin (1) and amycolose (2).

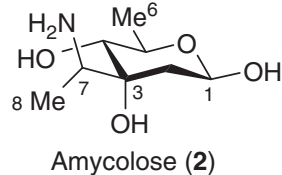

Amycolose (2) 


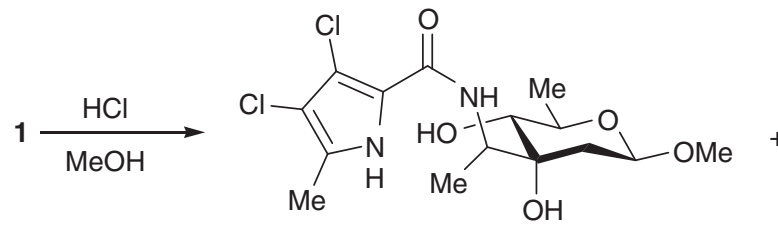

$\beta$-isomer (3) 64\%

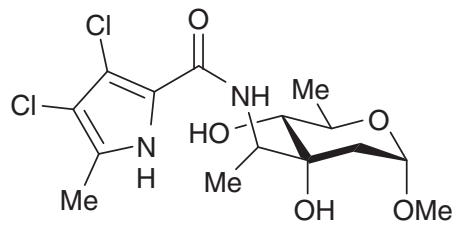

$\alpha$-isomer (4) 33\%

Scheme 1 Methanolysis of amycolamicin.
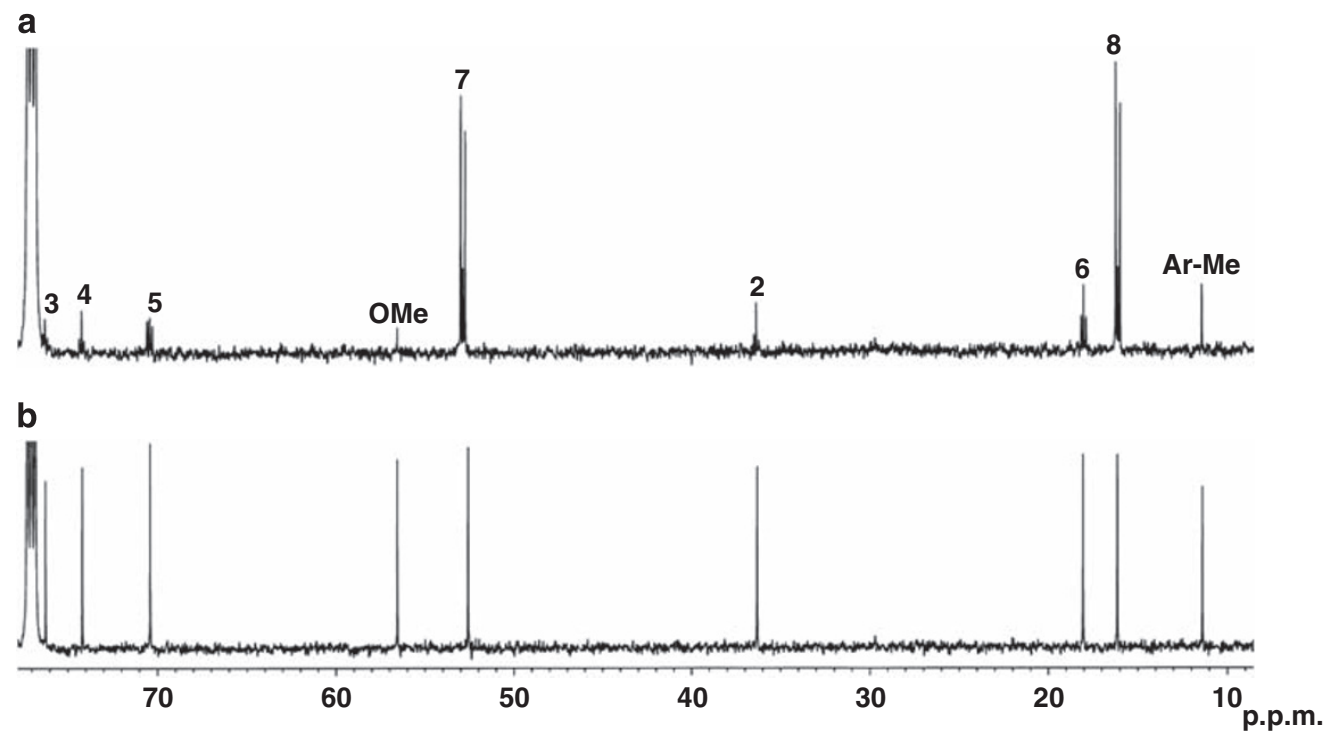

Figure 2 Partial ${ }^{13} \mathrm{C}$ NMR spectra (150 MHz, CDCI3). (a) Labeled amycolose derivative (3). Numbers show positions. (b) Unlabeled standard.

purified by silica gel column chromatography to give pure ${ }^{13} \mathrm{C}$-labeled amycolamicin (1.24 mg).

Amycolamicin shows extremely complex NMR spectra because of the presence of tetramic acid as a mixture of four tautomers. ${ }^{18,19}$ Consequently, to prepare an amycolose derivative that would show clear NMR spectra, methanolysis was carried out using unlabeled $\mathbf{1}$. By treating 1 with hydrogen chloride in methanol, amycolose derivatives retaining an amide bond ( 3 and 4 ) were obtained (Scheme 1). These derivatives show simple NMR spectra, and the NMR signals could be distinguished (Supplementary Information). Labeled amycolamicin was treated under the same conditions to prepare a labeled $\beta$-anomeric derivative (0.24 mg).

As shown in Figure 2, the ${ }^{13} \mathrm{C}$ NMR spectrum of the resulting labeled amycolose derivative clearly indicated strong incorporation at C-7 $(\delta$ 52.6) and C-8 $(\delta$ 16.1), in which spin coupling was clearly observed $\left({ }^{1} J_{\mathrm{CC}}=36.5 \mathrm{~Hz}\right)$. In the ${ }^{1} \mathrm{H}$ NMR spectrum (Figure 3), a double double doublet

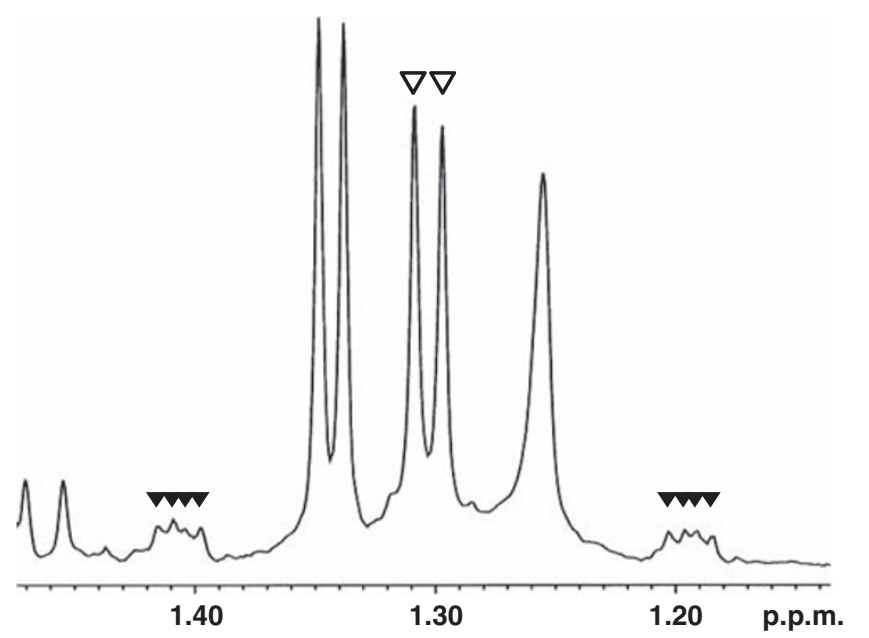

Figure 3 Partial ${ }^{1} \mathrm{H}$ NMR spectrum $(600 \mathrm{MHz}, \mathrm{CDCl} 3)$. The outline triangles show the doublet signal of Me-8 in the unlabeled amycolose derivative (3). The black triangles show the double double doublet signal of Me-8 in labeled amycolose derivative.

methyl signal $\left({ }^{1} J_{\mathrm{CH}}=125 \mathrm{~Hz},{ }^{2} J_{\mathrm{CH}}=4.1 \mathrm{~Hz}\right.$, ${ }^{3} \mathrm{~J}_{\mathrm{HH}}=6.9 \mathrm{~Hz}$ ), due to the $8-\mathrm{Me}$ of the labeled derivative, was observed, in addition to the doublet methyl signal $\left({ }^{3} \mathrm{~J}_{\mathrm{HH}}=6.9 \mathrm{~Hz}\right)$ of the unlabeled derivative. These results strongly indicated that positions 2 and 3 of pyruvate were incorporated intact into the branched $\alpha$ aminoethyl moiety of amycolose and revealed that the branched $\alpha$-aminoethyl moiety was derived from pyruvate. In addition, weak 


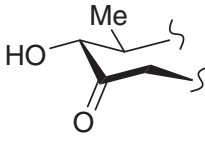

ketone precursor

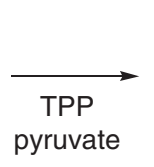

pyruvate

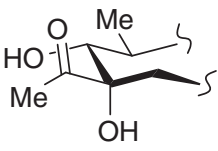

oxoethyl branch

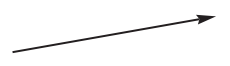

reductase

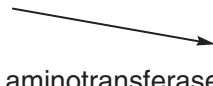

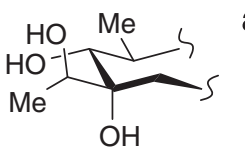

hydroxyethyl branch

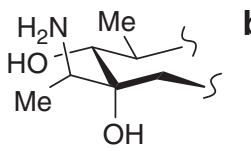

aminoethyl branch

\section{b}

Scheme 2 A possible biosynthetic pathway of the two-carbon branch.

intact incorporations at the main chain (C-2, 3, 4, 5 and 6) of amycolose could be explained in terms of gluconeogenesis and the pentose phosphate cycle. These incorporations could be observed when the compounds involved in the glycolitic system were fed. $^{20}$

In this study, we used a feeding experiment with ${ }^{13} \mathrm{C}$-labeled pyruvate to show that pyruvate was the precursor of the branched $\alpha$-aminoethyl moiety of amycolose, as well as other two-carbon branches. The attachment of two carbons is catalyzed by the thiamine pyrophosphate (TPP)-dependent enzyme, which catalyzes the formation of hydroxyethyl-TPP from pyruvate as a nucleophilic two-carbon donor and allows nucleophilic attack on the ketone at the main sugar chain to produce a branched $\alpha$-oxoethyl sugar. Then, ketone reduction occurs to form a hydroxyethyl branch (Scheme 2, route a). However, in the formation of a branched $\alpha$-aminoethyl, the aminotransfer of a ketone group to an amino group is the most probable reaction in amycolose biosynthesis (Scheme 2, route b). It remains unclear whether the accepter is the sugar nucleotide or the glycoside with the aglycone during the two-carbon chain formation. According to our recent research, genes encoding a TPP-dependent enzyme and a pyridoxal 5'-phosphate-dependent aminotransferase were found in the amycolamicin biosynthetic gene cluster. The products of these genes possibly catalyze the construction of the branched $\alpha$-aminoethyl moiety in the amycolose structure.

\section{ACKNOWLEDGEMENTS}

We thank Mrs Y Kubota and Dr R Sawa for measuring the NMR spectra and HR ESIFT-MS, respectively.

\section{Shigehiro Tohyama, Yoshikazu Takahashi and Yuzuru Akamatsu}

Microbial Chemistry Research Center, Microbial Chemistry Research Foundation, Shinagawa-ku, Tokyo, Japan E-mail: tohyamas@bikaken.or.jp

1 Igarashi, M. et al. Amycolamicin: a novel antibiotic from Amycolatopsis sp Abstr. 49th Intersci. Conf. Antimicrob. Agents Chemother., San Francisco 194 (F1-1497) (2009).

2 Igarashi, M., Sawa, R. \& Homma, Y. Jpn. Kokai Tokkyo Koho (2009) JP2009-203195.

3 Walsh, F. M. \& Amyes, S. G. Microbiology and drug resistance mechanisms of fully resistant pathogens. Curr. Opin. Microbiol. 7, 439-444 (2004).

4 Murray, B. E. Drug therapy: vancomycin-resistant enterococcal infections. New Engl. J. Med. 342, 710-721 (2000).

5 Griffith, B. R., Langenhan, J. M. \& Thorson, J. S. 'Sweetening' natural products via glycorandomization. Curr. Opin. Biotechnol. 16, 622-630 (2005).

6 Finkelstein, J. Glycochemistry \& glycobiology. Nature 446, 999 (2007).

7 Thibodeaux, C. J., Melançon, C. E. \& Liu, H.-w. Unusual sugar biosynthesis and natural product glycodiversification. Nature 446, 1008-1016 (2007).
8 Grisebach, H. \& Schmid, R. Chemistry and biochemistry of branched-chain sugars. Angew. Chem. Int. Ed. Engl. 11, 159-173 (1972).

9 Yoshimura, J. Synthesis of branched-chain sugars. Adv. Carbohydr. Chem. Biochem. 42, 69-134 (1984).

10 Grisebach, H. Biosynthesis of sugar components of antibiotic substances. Adv. Carbohydr. Chem. Biochem. 35, 81-126 (1978).

11 Liu, H.-w. \& Thorson, J. S. Pathways and mechanisms in the biogenesis of novel deoxysugars by bacteria. Annu. Rev. Microbiol. 48, 223-256 (1994).

$12 \mathrm{He}$, X., Agnihotri, G. \& Liu, H.-w. Novel enzymatic mechanisms in carbohydrate metabolism. Chem. Rev. 100, 4615-4661 (2000).

13 Schmid, R., Grisebach, H. \& Wolfgang, K. Zur Biosynthese der D-Aldgarose. Eur. J. Biochem. 14, 243-252 (1970).

14 Matern, U. \& Grisebach, H. Studies on the biosynthesis of the branched-chain sugars from the quinocycline complex. Eur. J. Biochem. 29, 5-11 (1972).

15 Matern, U. \& Grisebach, H. Bildung eines oxoäthylverzweigten Zuckers aus Thymidindiphosphatglucose und Pyruvant mit einem zellfreien. N. Naturforsch. 29, 407-413 (1974).

16 Chen, H., Guo, Z. \& Liu, H.-w. Biosynthesis of yersiniose: attachment of the two-carbon branched-chain is catalyzed by a thiamine pyrophosphate-dependent flavoprotein. J. Am. Chem. Soc. 120, 11796-11797 (1998).

17 Treede, I. et al. Genes involved in formation and attachment of a two-carbon chain as a component of eurekanate, a branched-chain sugar moiety of avilamycin A. Appl. Environ. Microb. 71, 400-406 (2005).

18 Steynnext, P. S. \& Wessels, P. L. Tautomerism in tetramic acids: ${ }^{13} \mathrm{C}$ nmr determination of the structures and ratios of the tautomers in 3-acetyl-5-isopropylpyrrolidine-2,4-dione. Tetrahedron Lett. 19, 4707-4710 (1978).

19 Nolte, M. J., Steyn, P. S. \& Wessels, P. L. Structural investigations of 3-acylpyrrolidine-2,4-diones by nuclear magnetic resonance spectroscopy and X-ray crystallography. J. Chem. Soc. Perkin 1, 1057-1065 (1980).

20 Takaishi, M., Kudo, F. \& Eguchi, T. Biosynthetic pathway of 24-membered macrolactam glycoside incednine. Tetrahedron 64, 6651-6656 (2008).

Supplementary Information accompanies the paper on The Journal of Antibiotics website (http://www.nature.com/ja) 